\title{
Forum
}

\section{Rationale for ongoing radio-collaring of black rhinos - a response to Alibhai and Jewell}

\author{
Raoul du Toit
}

\begin{abstract}
Alibhai and Jewell (this issue) list what they regard as five contra-indications to routine radio-collaring; their list can be used as a basis for presenting alternative perspectives that stem from the radio-collaring experience in Zimbabwe.
\end{abstract}

Their first point concerns the financial expenditure associated with radio-collaring. Alibhai and Jewell do not present any information to support their conclusion that radio-telemetry of rhinos is financially impractical in developing countries. Radio-telemetry is certainly expensive, but so are many other essential aspects of rhino management and protection, and major donor support is often required to effectively protect rhinos in range states such as Zimbabwe. So, accepting that radiocollaring is costly, we have to go on to ask whether it is nonetheless cost-effective as a tool for rhino monitoring in certain circumstances.

In Zimbabwe, relevant circumstances have arisen in the 'intensive protection zones' (IPZs) at Sinamatella and Matusadona. These large IPZs, each over $1000 \mathrm{sq} \mathrm{km}$, contain relatively small rhino populations ( $<100$ animals), and are unfenced portions of larger protected areas. Manpower resources are insufficient to provide blanket coverage at an 'intensive' level, and manpower therefore has to be deployed in accordance with information on the current distribution of the rhinos. The circumstances in small, fenced areas, or in larger areas with higher rhino densities or with higher manpower levels, would not generally justify routine radio-collaring.

If, as is stated by Alibhai and Jewell, the scale of radiocollaring in Zimbabwe is 'unusual' this is simply because of these different circumstances and not because other range states hold fundamentally different views on the circumstances that would justify radiocollaring. Indeed, there is currently a project within the Southern African Development Community (SADC) Rhino Programme (a regional capacity-sharing programme that involves all the southern African range states) to undertake ongoing trials of rhino radio-collars.

Raoul du Toit World Wide Fund for Nature, PO Box CY1409,

Causeway, Zimbabwe. E-mail: rdutoit@wwf.org.zw

Manuscript accepted for publication 6 June 2001
The cost-effectiveness of collaring has to be considered in the context of the overall protection system that can be established in an area with available resources. It is the view of Zimbabwe's wildlife authorities and donors that radio-collaring does make IPZ anti-poaching systems more efficient and economical. This enhancement is apparent even when only a proportion of the rhinos are radio-collared; to collar all rhinos or even most of the rhinos in an IPZ has not been feasible in the long-term. For instance, 25 rhinos are due to be immobilized at Sinamatella this year, of which only 10 will be selected for collaring, according to criteria such as how difficult they are to monitor and whether they tend to inhabit areas that are relatively less safe than other parts of the IPZ.

The fact that the number of rhinos to be immobilized is greater than the number that will be radio-collared illustrates another point that Alibhai and Jewell overlook, which is that rhinos are often immobilized for reasons other than radio-collaring. Immobilization costs and any veterinary risks are usually justified not only by the importance of radio-collaring but also because other monitoring needs are concurrently attended to. In particular, ear-notching has proven to be an essential component of rhino monitoring throughout southern Africa, not only to enable all rhinos to be accounted for in small populations but also to make it possible to estimate the sizes of larger populations using a markrecapture technique (Emslie \& Brooks, 1999). In addition, injectable short-range transponders are inserted under the skin of each rhino, and often into the horns, to ensure that the identities of dead rhinos can be confirmed even at advanced stages of decomposition.

Alibhai and Jewell outline animal welfare concerns as their next 'contra-indication'. The lesion shown in their photographs is one of the worst of the relatively few lesions that are known to have resulted from radiocollaring rhinos, but this rhino recovered fully, without need for further veterinary attention, after the collar had been removed. Even if more convincing evidence could be presented to support the suggestion that rhinos with collar lesions attract hyaenas Crocuta crocuta, this would not confirm that these minor lesions (equivalent to the natural filarial lesions on rhinos in humid areas) somehow make the rhinos, or their calves, more vulnerable to attack by hyaenas. 
Although Alibhai and Jewell have not presented an objective assessment of the animal welfare concerns associated with radio-collaring, this certainly does not mean that such concerns do not exist. Indeed, it is because of these concerns that the Zimbabwean rhino managers have tended to use collars that are easily shed, but create a low risk of injury if they stay on. The elaboration of 'hose collar' designs was discontinued not merely because they had a higher drop-off rate but because they posed a greater risk of snagging rhinos; the potential durability of the materials was greater than the battery life of the transmitters, which is clearly inadvisable.

Notwithstanding the continual need to consider animal welfare issues, rhino radio-collaring must be viewed in the context of research and development. There would have to be very convincing reasons to abandon the goal of developing a durable and 'rhino friendly' radio-collar, given the obvious potential to improve rhino protection through conventional radiotelemetry. Also, we might well take advantage of new developments in transponder and Global Positioning System technology. Despite the rapid reduction in the size of such devices, and despite the problems that arise with radio-collars on rhinos, other options to fit these devices to rhinos remain limited.

Because of veterinary considerations that apply particularly to rhinos, the use of body implants is not a definite option for these animals, in contrast to certain carnivores, and horn implants are no more durable than the current collar designs. Therefore, in view of potential benefits to rhino conservation, cautious research and development of rhino collars should not necessarily be suspended because of initial setbacks and costs, just as the development of rhino immobilizing drugs had to involve a certain amount of trial-and-error (including a significant mortality rate, which should not be the case with radio-collaring) in order that rhino translocation operations could become feasible.

The animal welfare issue is closely related to the fourth 'contra-indication' suggested by Alibhai and Jewell, which is that radio-collaring compromises female fertility. The suggestion that chemical immobilizations may disrupt rhino reproduction is far less emphatic than the initial assertion by Alibhai et al. (1999) that they had conclusive statistical proof that the fertility of female black rhinos at Sinamatella was 'significantly compromised' through drug immobilizations.

This controversial assertion gave rise to major concern, and so the IUCN African Rhino Specialist Group convened a working group to investigate the issue in May 2000. The conclusion was that the information that was available from Alibhai and Jewell did not support their allegation that rhino reproductive performance had been impaired. Indeed, the Sinamatella population had increased at a rate of 9-10 per cent per annum over the past 5 years, which is one of the highest sustained growth rates recorded for African rhinos. Not only does this exceptional reproductive performance suggest that drug immobilizations have not compromised rhino breeding, but it also suggests that any additional adverse effects of radio-collaring cannot be as serious as implied by Alibhai and Jewell.

The final 'contra-indications' that are stated by Alibhai and Jewell deal with the allocation of manpower. One 'contra-indication' is that radio-collaring may induce a false sense of security because of a concentration of monitoring effort on collared rhinos, while the other 'contra-indication' is that follow-up monitoring of collared rhinos is not likely to be maintained.

Apart from being somewhat contradictory, these points are speculative. On the basis of their experience with radio-collaring, the IPZ officers charged with the responsibility of rhino protection want this system to be continued and refined as a 'real-time' monitoring tool. As previously mentioned, the rhinos that are selected for radio-collaring are those that justify a concentration of monitoring effort, and for every rhino collared it is often possible to keep track of one or more other rhinos that associate with the collared individual.

Whereas Alibhai and Jewell are emphatic that attempts to develop new collar designs are doomed to failure, the fundamental problems are already wellknown (du Toit, 1996) and the prevailing view in southern Africa is that it should be possible to solve them through ongoing cautious experimentation. Time will tell which attitude is correct.

\section{References}

Alibhai, S.K., Jewell, Z.C. \& Towindo, S.S. (1999) The Effects of Chemical Immobilisation on the Fertility of Female Black Rhino (Diceros bicornis). Report to the Department of National Parks and Wildlife Management, Zimbabwe.

Emslie, R. \& Brooks, M. (1999) African Rhino: Status Survey and Conservation Action Plan. IUCN, Gland, Switzerland.

du Toit, R.F. (1996) Modern technology for rhino management. Pachyderm, 22, 18-24.

\section{Biographical sketch}

Raoul du Toit has been implementing rhino conservation projects for the World Wide Fund for Nature in Zimbabwe for the past 13 years, and has also been involved in rhino conservation activities in several other African countries. This work has ranged from basic rhino management activities (translocations, monitoring, anti-poaching support, etc.) to the development of land-use and community programmes that provide an enabling environment for long-term rhino conservation. 